\title{
Adoption of Disabled Children in Developed Countries: Practice, Research and Its Reference for China
}

\author{
Bo Wang \\ College of Early Childhood and Special Education, Weifang, Shandong, China
}

\begin{abstract}
With the social progress, development of children adoption, and improvement of views on the disabled, adoption of disabled children has gradually attracted social attention. This paper analyzed the practice and research of regulations and policies, preparation, support systems in developed countries, in order to provide reference for the Adoption of Disabled children in China.
\end{abstract}

Keywords - disabled children, adoption, the best interests of the child, support system

\section{发达国家残疾儿童收养的实践、研究与中国借鉴}

\author{
王波 \\ 潍坊学院幼教特教师范学院, 潍坊, 山东, 中国
}

\begin{abstract}
摘 要 随着社会进步、儿童收养事业发展以及残疾人观的改善, 残疾儿童收养逐渐进入社会关注的视野。文章梳理了发达国 家在残疾儿童收养法规、残疾儿童收养的准备、支持体系等方面的实践与研究，以期对我国残疾儿童收养提供借鉴和参考。

关键词＼cjkstart残疾儿童，收养，儿童最佳利益，支持体系
\end{abstract}

残疾儿童是身心发展上有各种缺陷的儿童, 包括智力 残疾、听力残疾、视力残疾、肢体残疾、言语障碍、情绪 和行为障碍及多重障碍等 $[1]$ 。近些年来, 随着社会进步、 儿童收养事业发展以及残疾人观的改善, 残疾儿童收养逐 渐进入社会关注的视野。与普通儿童相比, 残疾儿童由于 其自身的缺陷和障碍, 更难被收养安置, 并且, 他们给养 父母带来的挑战更大, 需要高水平的专门性教育、以及心 理和医学服务。目前, 美英等发达国家均建立了完善的法 规政策, 在残疾儿童收养方面积累了丰富的经验, 相关研 究也较为成熟。发达国家残疾儿童收养的先进经验做法, 可为我国残疾儿童收养提供借鉴和参考。

\section{1.残疾儿童收养的政策法规}

收养立法是亲属立法的重要组成部分。完善、严谨的 收养立法, 不仅有利于收养关系的建立与稳固, 也有利于 收养冲突的调适与规范[2]。为满足包括残疾儿童在内的特 殊需要儿童的收养需求, 美英等许多发达国家颁布了完备 的收养政策法规和配套政策法规体系。例如美国相继颁布 了《收养救助和儿童福利法案》(Adoption Assistance and
Child Welfare Act）、《霍华德 $\mathrm{M} ・$ 梅岑鲍姆多民族安置法案》

(Howard M. Metzenbaum Multiethnic Placement Act) 和《收 养和安全家庭法案》(Adoption and Safe Families Act) 等法 案; 加拿大于 1996 年颁布实施了《收养法案》(Adoption Act 1996), 该法案对收养准备程序、法院审理程序、违规与惩 罚等 9 大条款做了详细规定; 英国在吸收《1989 儿童法》 （Children Act 1989）和 1993 年海牙《跨国收养方面保护 儿童及合作公约》确定的儿童最佳利益原则、并继承以往 立法经验的基础上, 于 2002 年通过了《2002 收养与儿童 法》(Adoption and Children Act 2002)。作为“改变收养制度 面貌的一部法律”, 该法律在收养功能、收养原则和收养具 体制度上进行了新的变革。

1989 年联合国儿童权利公约 (UN Convention on the Rights of the Child）中明确了“儿童最佳利益”（the best interests of the child）作为各国亲子法所遵循的最高立法原 则, 确立了儿童在亲子法中的主体性。为使养子女福祉得 到更好地保护, 近些年来, 许多国家的收养政策法规均以 养子女的利益作为首要考虑因素。在加拿大的《收养法案》 中, 第一条款的第三项列举了在决定儿童最佳利益时必须 
考虑的相关因素, 包括（1）儿童安全; (2) 儿童身体和情 绪的需求与发展水平;（3）持续抚养儿童的重要性; (4) 与父母有良好关系、以及作为家庭一员的安全感, 对儿童 的发展有重要影响; (5) 该儿童与父母和他人关系的质量, 以及维持该关系带来的影响; (6) 儿童的文化、种族、语 言和宗教的继承; (7) 儿童的意见; (8) 如果需要延迟做 决策, 对儿童产生的影响[3]。

\section{2.残疾儿童收养的准备}

残疾儿童收养是一项系统工程, 需要做好充分的收养 准备, 与安置到福利机构相比, 残疾儿童收养计划的实施 往往耗费时间更多。美国健康和人类服务部总监办公室的 报告显示, 1991 年美国 20 个州的残疾儿童从接受寄养 (foster care) 到实施收养平均耗时 3.5 年到 5.5 年。在美 国, 残疾儿童从寄养系统到收养一般需要经历三个阶段:第 一个阶段是重组阶段 (reunification), 第二个阶段是收养计 划准备阶段, 包括亲生父母养育权利的剥夺, 第三个阶段 是收养计划阶段 [4]。在波兰, 个人收养儿童所耗费的时间 也比较长, 例如在 2012 年新的收养法实施之前, 收养儿童 的程序一般要耗时 3-4 年的时间; 在加拿大安大略省, 从 儿童福利机构到收养的过程也耗时较长, 从开始的正式申 请到安置到家庭接受强制性试收养 (pre-adoption) 一般需 要 24 个月。在这一复杂过程的早期阶段, 收养申请人必须 完成一系列的申请, 以正式表明其收养兴趣, 通常申请人 要填写偏好表格, 以表示愿意接受残疾孩子的潜在意愿; 中间阶段, 申请人参加一系列教育会议并参加收养工作人 员举办的几届家庭研习。如果在司法管辖区内正式获得了 收养权, 申请人就能被指派一名收养工作者。申请人与指 定的收养工作人员取得联系后进入第三阶段。为达到残疾 儿童试收养的目的, 双方进一步探讨他们的能力和儿童的 偏好特征, 以促成儿童需要与和申请人意愿之间的匹配。 并且中晚阶段之间, 申请人往往有一个相当长的等待期 (例 如 3-9 个月), 在这期间, 申请者必须不断评估是否继续抑 或退出[5]。

研究发现, 养父母的准备度有利于形成将来与养子女 的关系, 并对儿童行为、家庭生活、较低的父母压力等方 面的正面评价有重要影响, 因此, 对残疾儿童养父母的准 备度进行评估具有重要意义[6]。Egbert 和 LaMont 采用定 性分析和定量分析方法, 就影响其收养准备的因素对 368 名养父母进行研究, 结果发现, 有 12 个关于儿童、家庭和 机构的变因与收养准备有关。儿童的归属能力、父母与机 构的联系、收养的持续性、父母在收养期间的年龄等能预 测父母对收养准备的感知[7]。Rosenthal 和 Groze 等针对收 养前准备阶段所接受的服务, 调查了 487 名养母和 75 名养
父, 结果显示三分之一的家庭希望了解他们收养孩子的更 多信息。特别是, 养父母们表示需要更多关于亲生父母的 背景信息、受虐待的历史以及医疗信息。对收养残疾儿童 特定障碍的相关知识的缺乏是养父母最大的忧虑之一 [8]; 而 Reilly 和 Platz 对美国内华达州 249 个家庭调查发现, 58\% 的家庭并没有获得足够的信息 [9]; Peterson 和 Freundlich 则强调了为养父母准确提供儿童健康和分娩史的书面信息 的重要性。根据家庭需要提供详尽的准确信息, 能协助机 构招募及挽留收养家庭, 以及帮助领养父母建立恰当的期 望值[10]。

为保证收养安置的效果和收养的稳定性、并促进残疾 儿童的身心健康, 应充分考虑养父母的收养意愿, 并对父 母收养意愿的进行评估。然而, 目前对养父母收养意愿的 评价工具相对较少, 与特殊需要儿童相关的项目缺乏, 导 致无法正确地评价收养意愿并提供针对性指导。为准确高 效低评估收养特殊需要儿童（尤其是残疾儿童和接受特殊 医疗的儿童）的养父母的收养意愿, 帮助指导他们的收养 决策, 使特殊需要儿童的收养需要与养父母的收养意愿想 吻合, Orme 等新开发了量表一《收养意愿量表一障碍和医 疗条件》 (Willingness to Foster Scale-Disabilities and Medical Conditions, WFS-DMC)。WFS-DMC 量表包含了 19 个项目条款, 针对 19 种障碍儿童和有医疗需要的儿童 的收养意愿进行评估。以全国的 298 名养母作为样本, 对 该量表进行测试, 结果显示其内部一致性信度为优秀 ( $a=.90)$ 。在结构效度方面, 研究发现, 具有较高 WFS-DMC 分数的母亲的抚养时间越长、收养和安置的儿童更多, 并 且要求解除儿童收养关系的比例较小[11]。

\section{3.残疾儿童收养的影响}

收养作为儿童安置的一种重要方式, 具有较长的历史, 并对儿童发展产生积极影响。来自经济合作组织成员国、 以及黎巴嫩和印度等国的研究均得出了较为相似的结果, 即与安置在孤儿院或寄养家庭（foster home）中的兄弟姐 妹或同伴相比, 被收养儿童取得的智商分数更高, 学业成 就更优秀, 并且行为问题更少[12]。目前对被收养人收养成 效的研究主要集中于以下几个方面：（1）肢体发展;（2） 自尊与认同; (3) 认知发展、学校成就和特定能力; (4) 心理调整, 包括心理健康和精神病理学; (5) 与父母（包 括依恋) 以及同伴的关系[13]。其中, 诸多研究表明, 收养 残疾儿童对家庭的影响总体较为肯定。Rosenthal 和 Groze 等对 780 名收养残疾儿童的养父母的调查发现, 三分之二 的养父母表示收养对家庭的影响比较积极 (mostly positive) 或非常积极 (very positive), 并且绝大多数家长对亲子互 动持较为肯定的态度[14]; Glidden 和 Valliere 等人研究发 
现, $62 \%$ 的受访母亲反映他们变得更好, 他们更加宽容、 自私心不那么强, 更富有同情心和共情能力[15]; Glidden 采用半结构化访谈和自陈式问卷, 对收养发展障碍儿童的 87 名母亲与家庭功能进行了研究, 评估了 9 个预测因子和 4 个结构变量间的关系。结果显示, 母亲对收养残疾儿童 总体表示出高满意度, 特别是, 家庭对残疾儿童安置的初 始反应和大约 5 年后的调适呈现正相关。自陈报告显示最 好结果是在安置初期母亲的预约较少、没有抑郁情况、已 婚、对残疾状况有丰富经验、并有较强的宗教信仰 [16]; Orme 和 Cherry 等对全美 304 名收养残疾儿童的养母的收 养意愿进行调查, 结果发现 $51 \%$ 的养母愿意无条件地收养 任何类型的残疾儿童 (除了艾滋病病毒感染儿童和艾滋病 患儿)。并且, 无条件收养残疾儿童的养母们的收养时间更 长、收养的儿童更多[17]。有的父母甚至通过收养几个残疾 儿童, 获得了较高的满意度。

近些年来, 诸多研究显示发展性障碍儿童的收养结果 往往比较乐观, 对家庭多会产生积极影响。相比情绪障碍 儿童, 发展性障碍儿童的收养中断率更低。一项针对收养 智力障碍、自闭症、脑瘫和和唐氏综合征儿童等发展性障 碍儿童的 42 个英国家庭的研究发现, 收养安置 3 年多之后, 在关于“如果还能做一遍, 你们是否还会收养? ”的问项中, $95 \%$ 的母亲给予了肯定回答, 这其中 $61 \%$ 的母亲表示收养 比他们预期的要好 [18]。然而与其他发展性障碍儿童相比, 有研究发现养父母更倾向于收养唐氏综合症儿童。究其原 因, 首先, 关于唐氏综合症儿童的信息充足, 对其诊断更 清楚确定; 其次是唐氏综合症儿童的支持和拥护团体的数 量更多, 父母更容易获得他们的支持帮助; 再次是媒体对 唐氏综合症儿童的普及宣传较多[19]。

\section{4.残疾儿童收养关系的中断}

残疾儿童的收养行为受多种因素的影响, 父母信仰、 情感、人口学特征都可能影响他们的收养决定。Deiner 和 Wilson 等研究发现, 许多收养残疾儿童等特殊需要儿童的 收养家庭成员年龄较大、并且在收养时期已婚、白种人、 中产阶级[20]。Goetting 等人对收养严重发展性障碍儿童的 养父母进行研究, 从人口统计学的角度来看, 样本中父母 的年龄普遍较大 ( $M=44.4$ 年), 大多是白人并且已婚, 有 超过 3.5 万美元的收入, 亲生孩子具有特殊需要 (special need)[21]。当前, 大多数关于残疾儿童收养的研究主要关 注于两方面的结果, 即收养持续性以及父母满意度。其中, 收养持续性是通过收养中断率 (disruption rate) 来测量的。 尽管收养相对比较稳定和成功, 但是有相当大比例的残疾 儿童收养出现中断或替代安置现象, 统计显示, 有大约 10-16\%的特殊需要儿童的收养出现中断现象, 也就是合法
化前后收养结束[22]。Coyne 和 Brown 对整个北美地区收 养的 1588 名发展性障碍儿童进行研究发现, 发展性障碍儿 童的收养中断率为 8.7\%[23]。而澳大利亚的一份研究显示, 有四分之一的智力障碍儿童收养以失败告终[24]。

许多与儿童有关的危险因素都可能导致收养中断, 但 是研究中最经常提到的因素包括收养年龄较大、儿童情绪 或行为问题。而多个预收养的危险因素往往同时出现, 包 括收养年龄较大、受虐待的历史、已经有问题行为和情绪 问题的家庭外安置 (out-of-home placement)。在导致收养 关系中断的因素中, 儿童的问题行为是经常被提及的。

Nalavany 和 Glidden 等对参加佛罗里达项目 (Florida Adoption Projcet,FAP)的 1865 名养父母的满意度进行调查, 结果显示收养学习障碍儿童的养父母的最大障碍不是来自 于儿童学习困难本身, 而是儿童行为问题[25]。Coyne 围绕 阻碍收养的行政因素的研究发现, 阻碍发展性障碍儿童成 功收养的不是其自身特征, 而是影响安置的机构政策、氛 围和体系[26]; Rosenthal 综合研究发现, 导致收养中断的 危险性增加的主要因素包括: (1) 在收养安置时年龄较大;

（2）背景信息不足或不切实际的养育期待;（3）家庭功能 模式僵硬, 特别是父亲不参与养育任务; (4) 亲戚或朋友 给予的支持层次低;（5）收养之前有遭受身体虐待, 特别 是性虐待的经历; (6) 收养之前在精神科住院; (7) 发泄 性行为等外化行为问题; (8) 没有被养父母收养, 而是被 新的父母收养安置[27]。

\section{5.残疾儿童收养的支持保障体系}

成功的收养安置不仅需要国家政策法规提供制度保 障, 还需要收养家庭和养子女之间的“匹配” (match), 更 离不开来自于医院、社区等外界的支持。如果不能给养父 母提供充分准备, 并且在收养过程中缺少综合性支持服务, 往往导致收养关系的中断。因此, 对收养父母提供支持性 服务显得尤为重要。Marcenko 和 Smith 对 125 个收养发展 性障碍儿童的家庭获得支持服务的情况进行调查, 结果显 示, 尽管总体他们对服务的满意度较高, 但是许多家庭仍 然没有得到暂托护理（respite care）和生活规划等基本的家 庭支持服务[28]。暂托护理项目由受过专门训练的人员为残 疾儿童提供临时照看服务, 让养父母从繁忙的养育中暂时 解脱出来, 以减轻他们的压力, 并为从事其他活动提供闲 暇时间。暂托服务可以在家庭之内, 亦可以在家庭之外, 例如在照看者家中、日托中心、团体寄养中心、或者互相 提供暂托服务的互助家庭中[29]。缺乏暂托护理, 特别是残 疾儿童的暂托护理, 往往增加养父母的压力, 导致监护人 产生养育倦急, 甚至导致收养关系的解除。Hudson 和 Levasseur 的研究发现, 收养残疾儿童的养父母, 得到暂托 
护理后的心理压力明显降低[30]。因此, 定期地让养父母离 开收养的残疾儿童一段时间具有重要意义。而对于收养发 展性障碍儿童的养父母来说, 发展性障碍儿童的特点决定 了养父母的抚养需求往往是终生的, 因此, 对家庭的个别 化的持续支持显得尤为重要。Brown 和 Moraes 等以“什么 服务和支持对你有帮助? ”作为问题, 对加拿大的养父母进 行调查, 结果显示养父母们最需要的服务和支持主要有: 社区支持、财政支持、学校安置系统、与社工的良好关系、 易于共鸣的专业人士、信息、综合医疗服务、对土著家庭 和儿童的服务、转衔服务等[31]。

越来越多的研究证明, 在收养前的准备阶段, 组建一 个非正式的收养支持团体具有重要意义。团体准备可以在 筛查程序方面为领养父母提供支持, 帮助机构和家庭就收 养残疾儿童的适宜性进行评估, 让那些即将收养儿童的父 母讨论关于教养方面的问题和经验, 并建立恰当的收养期 望值; 同时, 支持团体对残疾儿童养父母有许多益处, 还 包括能养父母感受到被他人理解、习得新的养育技能、表 达自己情感和顾虑、以及对儿童的亲生父母产生共鸣[32]。 而在残疾儿童收养初期, 有经验的社工能为养父母提供高 效的支持服务。养父母眼中的社工素质包括: 对养父母的 兴趣和投入、热情、可靠、倾听能力、可信赖以及行政事 务处理能力[33]。特别是在儿童收养安置的最初几个月中, 社工的连续性、与养父母和养子女沟通交流的频率与密度 等最重要。另外, 作为对残疾儿童有影响的重要他人 (significant others), 对养父母进行关于残疾 (障碍) 方面 的相关培训具有重要意义, 对养父母进行的专门培训能影 响对残疾儿童需求方面的知识技能的习得。在美国, 准养 父母们收养儿童需要接受一定类型和数量的试收养培训 (preadoption training), 各州之间没有千篇一律的培训模 式, 例如有些州采用名为《父母作为疗愈师》(Parents as Tender Healers, PATH)、《信息、发展与教育之父母资源》 (Parent Resources for Information, Development, and Education, PRIDE) 、《养育合作者示范方法》 (Model Approach to Partnerships in Parenting, MAPP)等培训课程, 而有些州在课程之外加入了远程学习内容[34]。

\section{6. 发达国家残疾儿童收养的实践与研究对我国的借鉴}

\section{1 构建完备的收养政策法规体系}

完善的收养政策法规能确保残疾儿童的权益得到有效 保护, 推进残疾儿童收养工作的健康可持续发展。我国应 充分借鉴国内外经验, 突出儿童权益优先原则, 构建完备 的收养政策法规体系。在现有的相关法规政策中, 加入残 疾儿童收养的内容并使之具体化和可操作化。同时, 为发
挥收养政策法规的执行力, 应建立收养政策法规的各种配 套政策法规，包括地方制定适合地域特色的收养制度。2014 年 12 月 1 日我国开始执行的《家庭寄养管理办法》中就规 定, 符合收养条件, 有收养意愿的寄养家庭, 可以依法优 先收养被寄养儿童。目前, 上海市从本地实际出发, 结合 当前的收养需求和现状, 颁布实施了新版的《内地居民收 养上海市儿童福利院弃 (㚣) 儿须知》, 初步明确和规范了 “先评估、再配对、后试养、最后登记”的科学化的收养评 估流程, 有效避免了盲目送养导致的矛盾和问题。残疾儿 童收养的“上海模式”使收养评估与送养人选择收养人以及 办理收养登记程序有机融合、相互衔接, 值得其他地区学 习和借鉴。

\section{2 建立科学的收养评估制度和收养评估指标体系}

收养工作服务于残疾儿童, 应以科学发展观为指导, 贯彻“以人为本”的方针, 使残疾儿童利益最大化, 这需要 建立完善的收养评估制度, 并确定收养动机、收养胜任力 等科学的评估指标体系。我国目前已经基本确定评估范围 和内容, 但尚未建立具体全面的收养评估指标体系, 指标 较为含糊。而收养评估是对收养人抚养教育被收养人能力 的综合评定, 需确定科学的评估指标体系, 以确保评估结 果的可信度。除收养人的收养动机、经济状况、婚姻状况、 品德品行等指标外, 还要对被收养人情况以及影响收养人 抚养教育被收养人能力的其他因素进行深入研究, 努力将 其转化为可通过约见、走访等办法观察反映的客观指标, 并积极尝试运用其他方法对各项指标进行检验、完善, 在 实践中逐步建立科学、全面、开放的评估指标体系。另外, 在科学收养评估体系的构建中, 还应尝试开发本土化的养 父母收养意愿量表。

\section{3 完善跨国收养的支持系统}

跨国收养(涉外收养)是残疾儿童永久性安置的一种有 效形式, 也是儿童福利事业的有机组成部分。我国政府历 来高度重视儿童权益保护工作, 目前已与美国、西班牙等 17 个国家的 138 个政府部门和收养组织建立了收养合作关 系, 十多万名残疾儿童在内的孤残儿童通过涉外收养回归 了家庭, 享受到了养父母的关爱和家庭的温暖[35]。据统计, 涉外收养登记中残疾、大龄等特殊需要儿童比例呈上升趋 势。2012 年涉外收养中残疾、大龄等特殊需要儿童达 $74 \%[36]$ 。仅以南京市为例, 国外家庭领养的残疾儿童占到 福利院残疾儿童总数的 $40 \%$ 左右, 近 20 年有近千名残疾儿 童被这些国外家庭领养, 仅 2010 年被领养的残疾孩子就有 90 多名[37]。为此, 应该努力为跨国收养家庭提供支持, 
针对国外父母养育中国儿童的需要, 力所能及地提供政策 保障和专业服务。例如政府可提供特殊政策, 促进政府和 民间机构的参与收养支持。地方可以通过政府购买服务方 式, 引入社工介入收养服务, 开展对收养家庭的收养前培 训、调查评估和跟踪回访工作, 并且针对领养家庭在收养 决策、养父母心理健康等方面的不同需要, 提供全方位的 服务。为给残疾儿童收养事业发展等提供决策支持和技术 保障, 中国儿童福利和收养中心启动的全国儿童福利信息 管理系统就是一个很值得肯定的创举。

\section{4 树立正确的残疾观和收养观, 促进残疾儿童收养}

目前, 国内对儿童的收养需求很大, 但是愿意收养残 疾儿童的家庭较少。以上海为例, 每年提出收养申请的家 庭有一、二百个, 但明确表示愿意收养残疾儿童的家庭却 不足 $2 \%$ 。让人欣喜的是, 国人的收养理念正在悄然转变中, 不少收养意愿比较坚决的家庭开始逐渐考虑接受残疾儿童 [38]。为此, 应改变社会的固定观念, 增强对残疾的认识, 树立正确的残疾观, 促进对残疾儿童的收养。国外注重强 化社会对残疾人的正确认知, 强调从娃娃开始抓起的做法 值得我们学习。另外, 国家还应加大收养法律、法规的宣 传, 引入助人自助的社工理念, 让民众充分认识到收养所 需承担的责任和义务, 帮助他们端正收养动机、树立正确 的收养观念。

\section{参考文献(References)}

[1] Y.X. Piao, Special Education Dictionary (Second Edition), Beijing: China Press .2006.8.

[2] G.Y. Wang, Reflection and Reconstruction on Legislation of Adoption in China. Northern Forum .2000, 6: 655-658.

[3] Adoption

Act. http://www.bclaws.ca/civix/document/id/complete/statreg/9600 5_01

[4] McKenzie J K. Adoption of Children with Special Needs. The Future of Children, 1993, 3(1): 62-76.

[5] Burge P, Jamieson M. Gaining Balance: Toward a Grounded Theory of the Decision Making Processes of Applicants for Adoption of Children with and without Disabilities. The Qualitative Report, 2009, 14(4): 566-603.

[6] Sar B K. Preparation for adoptive parenthood with a special needs child: Role of agency preparation tasks. Adoption Quarterly, 2000, 3(4): 63-80.

[7] Egbert S C, LaMont E C. Factors Contributing to Parents'
Preparation For Special-Needs Adoption, Child and Adolescent Social Work Journal, 2004, 21(6): 593-609.

[8] Rosenthal J A, Groze V, Morgan J. Services for families adopting children via public child welfare agencies: Use, helpfulness, and need. Children and Youth Services Review, 1996, 18(1/2): 163-182.

[9] Reilly T, Platz L. Characteristics and challenges of families who adopt children with special needs: An empirical study. Children and Youth Services Review, 2003, 25: 781-804.

[10] Peterson L, Freundlich M. Wrongful adoption. Children's Voice, 2000, 1: 20-23.

[11] Orme J, Cherry D J, Cox M E. Measuring willingness to foster children with disabilities and special medical conditions. Social Work Research, 2013, 37(3): 169-178.

[12] Christoffersen M N. A Study of Adopted Children, Their Environment, and Development: A Systematic Review. Adoption Quarterly, 2012, 15: 220-237.

[13] Palacios J, Brodzinsky D. Adoption research: trends, topics, outcomes. International Journal of Behavioral Development, 2010, 34(3): 270-284.

[14] Rosenthal J M, Groze V,Aguilar G D. Adoption Outcomes for Children with Handicaps. Child Welfare, 1991, 6: 623-636.

[15] Glidden L M, Valliere V N, Herbert S L. Adopted children with mental retardation: Positive family impact. Mental Retardation, 1988, 26: 119-125.

[16] Glidden L M. Adopted children with developmental disabilities: Post-placement family functioning. Children and Youth Services Review, 1991, 13: 363-377.

[17] Orme J, Cherry D J,Krcek T E. Who Is Willing to Foster Children With Disabilities? Journal of Public Child Welfare, 2013, 7(5): 566-585.

[18] Glidden L M. Families adopting children with mental retardation. Journal of Children in Contemporary Society. 1990, 21: 177-205.

[19] Glidden L M, Cahill B M. Successful Adoption of Children with Down Syndrome and Other Developmental Disabilities. Adoption Quaterly, 1998, 1(3): 27-43.

[20] Deiner P, Wilson N, Unger D. Motivation and Characteristics of Families Who Adopt Children with Special Needs: An Empirical Study. Topics in Early Childhood Special Education, 1988, 8(2):15-29.

[21] Goetting A,Goetting M G. Adoptive parents to children with severe developmental disabilities: Aprofile. Children and Youth Services Review, 1993, 15: 489-506. 
[22] Barth R, Berry M, Yoshikami R, et al. Predicting adoption disruption. Social Work, 1988, 33: 227-233.

[23] Coyne A, Brown M E. Developmentally disabled children can be adopted. Child Welfare, 1985, 64(6): 607-615.

[24] Hockey A. Evaluation of adoption of the intellectually handicapped: A retrospective analysis of 137 cases. Journal of Mental Deficiency Research, 1980, 24: 187-202.

[25] Nalavany B A, Glidden L M, Ryan S D. Parental Satisfaction in the Adoption of Children with Learning Disorders: The Role of Behavior Problems. Family Relations, 2009, 58 (5): 621-633.

[26] Coyne A. Administrative and policy issues. In Glidden L, Frank M (Ed.).Formed families: Adoption of children with handicaps. NY: Haworth, 1990. 93-115.

[27] Rosenthal J A. Outcomes of Adoption of Children with Special Needs. The Future of Children, 1993, 3(1): 77-88.

[28] Marcenko M O, Smith L K. Post-adoption needs of families adopting children with developmental disabilities. Children and Youth Service Review, 1991, 13: 413-424.

[29] Lightfoot E, LaLiberte T. Serving Children with Disabilities in the Child Welfare System: Barriers and Strategies. Impact, 2005, 19(1): 8-9.

[30] Hudson P, Levasseur, K. Supporting foster parents: Caring voices. Child Welfare, 2002, 81: 853-877.
[31] Brown J D, Moraes S, Mayhew J. ervice needs of foster families with children who have disabilities. Journal of Child and Family Studies, 2005, 14(3): 417-429.

[32] Redding R E, Fried C, Britner P A. Predictors of placement outcomes in treatment foster care: Implications for foster parent selection and service delivery. Journal of Child and Family Studies, 2000,9425-447.

[33] O'Hara, G. Developing post-placement services in Lothian. Adoption \& Fostering, 1986, 10: 38-42.

[34] https://www.childwelfare.gov/adoption/adoptive/preadoption.cf $\mathrm{m}$

[35] China News Network. Chinese Foreign Adoptions for 30 Years: An Important Way to Promote the Placement of Orphans and Disabled children. http://www.chinanews.com/gn/2012/07-05/4009962.shtml

[36] M.1. Wei, 74\% of Foreign Adopted Children are Children with Special Needs. Xinhua Daily Telegraph, 2013-11-21.

[37] D.J. Ma, Jun. Jin, Disabled Child Adoption Appliers are Mainly Foreign Families. Nanjing Daily, 2012-10-04.

[38] People's Republic of China Ministry of Civil Affairs. Shanghai Actively Exploring Adoption Assessment Pilot. http://sws.mca.gov.cn/article/dfdt/201303/20130300427552.sht $\mathrm{ml}, 2014-11-12$ 